\title{
Quantitative optical microscopy of colloids: the legacy of Jean Perrin
}

\author{
Roberto Cerbino ${ }^{1}$ \\ University of Milan, Dep. of Medical Biotechnology and Translational Medicine, Via Fratelli Cervi 93, 20090 Segrate, Italy
}

\begin{abstract}
When the discontinuous structure of matter was yet an intriguing hypothesis, Jean Perrin performed a set of elegant and pioneering experiments that marked the birth of what today we consider quantitative optical microscopy. Picking up the baton from Perrin, today microscopists face incredible challenges, aiming to extract quantitative information from the increasingly content-rich and complex images made available by modern microscopy techniques. Here, I provide an overview of these challenges and describe the solutions adopted to succeed in this complex task when investigating colloidal systems or systems in which colloidal particles are embedded as microrheological probes.
\end{abstract}

\section{Introduction}

Jean Perrin (1870-1942) has been a pioneer of quantitative optical microscopy. Reading his 1909 report in the Annales de Chimie et de Physique

5 (English translation 1]) is a stimulating and rewarding experience for any perspective scientist, in particular for experimentalists. In his manuscript, he describes a set of experiments that were stimulated by Einstein's 1905 predictions on Brown10 ian motion 2. Using a microscope equipped with a camera lucida, Perrin and his collaborators observed colloidal suspensions of mastic and gamboge particles at constant temperature. At a fixed height within the sample, the particles' radius $R$ was esti15 mated and their position was tracked in time (every 30 seconds) to extract their diffusion coefficient $D$. Einstein theory, providing a quantitative link between $D$ and $R$, was then exploited by Perrin to estimate the Avogadro number. Perrin also re-

20 ports several other observations, including an independent estimate of the Avogadro number based on the determination of the equilibrium sedimentation profile of the colloids, the measurement of the rotational diffusion coefficient of large spherical

25 inclusions, and the onset of colloidal aggregation experiments performed by adding coagulants. The value of Perrin experiments was immediately recognized by his contemporaries. In a letter to Perrin

\footnotetext{
${ }^{1}$ roberto.cerbino@unimi.it
}

dated 1909, Einstein himself wrote: "Ich hätte es so für unmöglich gehalten, die Brownsche Bewegung so präzis zu untersuchen; es ist ein Glück für diese Materie, dass Sie sich ihrer angenommen haben.", which in English reads: "I would have thought it impossible to investigate Brownian motion so pre-

35 cisely; it is fortunate for this matter that you have embraced it.". While not the first to provide an estimate for the Avogadro number, the meticulous care put by Perrin in all his experiments provided a strong, irrefutable argument in favor of the molec-

40 ular reality of matter and, for this work in particular, he was awarded with the 1926 Nobel prize in Physics.

Retrospectively, we can today consider Perrin the first exponent of quantitative microscopy, intended ${ }_{45}$ in the present review article as the art of pushing microscopy to its limits of temporal and spatial resolution by combining a set of diverse competences and skills borrowed from different disciplines such as physics, biology and engineering. Given the com50 plexity and the multidisciplinary nature of this art, it is thus not surprising that over the years, the heritage of Perrin has been collected by scientists of different branches, whose work contributed to shape a vast and variegated landscape that in this 5 review article I will only be able to scratch.

The modern approach to quantitative microscopy originates from efforts that have been very discontinuous in time. Indeed, up to about twenty years ago, optical microscopy was used mainly in a qual- 

pointed out very effectively in the introduction of Ref. 3. In the last twenty years, by contrast, we witnessed to a striking resurgence of quantitative optical microscopy experiments, mostly in nology: microscopes have been pushed to unprecedented limits both in terms of optics (e.g. optical resolution enhancement) and of mechanics (e.g. fast automation, high-precision positioning, intecentral processing units (CPU) has increased constantly and conformed to Moore's law far beyond any reasonable expectations; the parallel use of graphics processing unit (GPU) made possible the

75 high-speed real-time or post-acquisition analysis of 125 microscopy data, with speed-up factors exceeding 100X; pixelated image sensors can now measure single photons per pixel, can reach $10 \mathrm{KHz}$ acquisition speed at full frame ( 1 megapixel) and can acquire more than $10^{6}$ images per second at reduced image resolution, an incredible set of advances since the first demonstration of the charge-coupled device (CCD) in 1969

In this review article, I will try to cover the po- ${ }_{135}$ tentially very wide area of quantitative optical microscopy of colloidal systems, for which the relatively large length-scale is accompanied by dynamics and kinetics that are slower, and in turn easier to monitor, compared to molecular systems. The ${ }_{140}$

90 article is organized as follows: after a short section sketching the main advantages of optical microscopy, I will briefly describe in Section 3 the most recent efforts to push quantitative optical microscopy beyond the known resolution limits: it will ${ }_{5}$ be discussed how super-resolution techniques have been used in colloidal science so far and also what are the current performances of particle tracking algorithms. In Section 4 the recently introduced idea that microscopes can be used as very powerful light ${ }_{150}$ 100 scattering machines will be described. In particular, I will show how microscope movies obtained by imaging a sample in real-space with one or more image contrast mechanisms (e.g. absorption, dephasing, scattering, fluorescence) can provide wave- 155 105 vector resolved scattering information about it. Finally, Section 5 will be devoted to the use of microscopy, possibly in combination with rheology, to link the macroscopic mechanical properties of colloidal crystals and amorphous solids to the struc- 160 110 tural and dynamical properties.

\section{Advantages of optical microscopy}

Optical microscopy is one of the several tools used to characterize colloidal systems. Others are for instance (scanning or transmission) electron microscopy and atomic force microscopy; static and dynamic scattering (or diffraction) of light, neutrons or X-rays; sedimentation, centrifugation, field-flow fractionation; optical and ultrasonic spectroscopy; electro- and thermo-phoresis; viscometry and rheology.

Compared to all these methods, optical microscopy has some characterizing advantages. With respect to neutron, electron or x-ray microscopy it allows accessing shorter time scales over typically longer experimental durations and larger length scales. The extracted real-space information is complementary to the one obtained from scattering methods that usually access smaller length-scales (especially with X-rays and neutrons) and shorter time-scales, owing to the use of fast and sensitive detectors such as photomultipliers or avalanche photodiodes. We will show that the existing dichotomy between microscopy and scattering can be partly reconciled by using the ideas described in Section 4

Microscopes can be very easily coupled with other tools, such as rheometers, optical tweezers, microfluidic chips. Also, typical samples for microscopy can be rather thin (down to a few tens of $\mu \mathrm{m}$ ) along the optical axis, which helps in reducing multiple scattering from optically dense colloidal systems and unwanted convective flows.

Light intensity in micrographs can map absorption or dephasing fluctuations within the sample, as well as the distribution and conditional activity of fluorophores or depolarizing structures, providing thereby an extremely rich portfolio of probes. The spatial and directional properties of the illuminating light can be matched to an arbitrary degree with the collection optics, which enables to range from a fully three-dimensional (3D) detection (e.g. bright field microscopy with coherent illuminations) to an almost two-dimensional (2D) one (e.g. confocal or light-sheet microscopy) and permits the use of sophisticated optical schemes such as phase-contrast, dark-field or differential-interference contrast (DIC) that, at least in commercial setups, can be combined with others and alternated in a simple and quick way.

Finally, science-grade microscopes are ubiquitous in research laboratories, either academic or in- 
dustrial, with an ample price range that can of- 210 fer a good match for almost every interested new adopter. Readers interested in knowing in more about their applications in soft matter science may refer to Refs. [4, 5].

\section{Pushing the limits of quantitative optical microscopy}

Some of the limitations of optical microscopy of colloids are technological in nature, such as the signal to noise ratio in microscope images, which is key in discriminating a colloidal particle from the background signal and in determining the particle localization uncertainty. Some others are of fundamental nature. For instance, according to classical linear optics, diffraction poses an ultimate limit to the detection of small colloidal particles. In this Section, I will discuss how both technological and fundamental limitations have been tackled in the last years, with particular reference to colloidal systems.

\section{Super-resolution studies of colloids}

When considering recent improvements in optical microscopy, the first thought goes to the so called super-resolution techniques, such as stimulated emission depletion (STED) [6], photo-activated localization microscopy (PALM) 7, and stochastic optical reconstruction microscopy (STORM) [8]. With these revolutionary techniques, some nonlinearities in the emission and/or in the detection of light are exploited to smash the diffraction barrier and achieve resolutions of the order of few tens of nanometers. For introducing such groundbreaking ideas, Eric Betzig, Stefan W. Hell and William E. Moerner shared the 2014 Nobel Prize for Chemistry. 220

It is quite curious that the first adopters of these methods were life scientists rather than chemists or physicists, despite the fundamental contribution of the latter in developing these tools. In particular, colloid scientists started using super-resolution 225 optical microscopy with a notable delay, which is partly justified by the fact that some of the most common super-resolution approaches require specialized fluorescent probes and/or are intrinsically limited in terms of temporal resolution.

One of the early application of STED to colloidal science was demonstrated in Refs. 9, 10, where (i) the crystalline structure formed by $200 \mathrm{~nm}$ large particles, with voids in the crystal as small as 30 $\mathrm{nm}$, was unveiled with unprecedented lateral (43 $\mathrm{nm}$ ) and axial $(125 \mathrm{~nm})$ resolution and (ii) the formation of the colloidal crystals was monitored with a temporal resolution of $5 \mathrm{~ms}$, which allowed visualizing the annealing of potential point defects. More

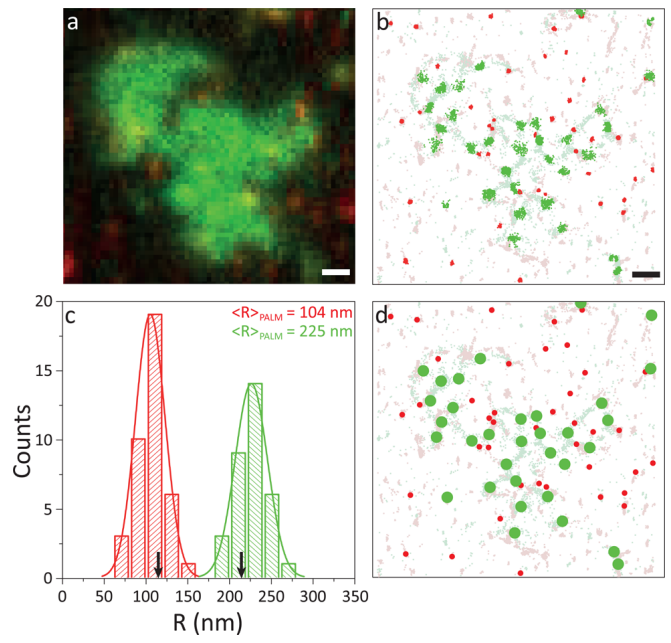

Figure 1: (a) Two-color wide-field image acquired in cyclohexane with $2 \% \mathrm{v} / \mathrm{v}$ isopropanol of a mixed cluster of colloidal particles of $R \sim 110 \mathrm{~nm}$ labeled with a red-emitting fluorophore and $R \sim 220 \mathrm{~nm}$ labeled with a green-emitting fluorophore. (b) Super-resolved image of the field of view shown in (a). Scale bar $1 \mu \mathrm{m}$. (c) Radii distribution of the nanoparticles identified and reconstructed in the mixed sample; black arrows indicate the mean radii determined by SEM.(d) Schematic representation of the beads identified in (b). Reprinted with permission from Ref. 11. Copyright (2016) American Chemical Society.

recently, Aloi et al. [1] demonstrated the possibility to perform high-accuracy super-resolution microscopy with various organic solvents by using PALM to obtain the size distribution of binary mixtures of nanoparticles in close contact. The usefulness of this approach can be appreciated in Fig. 1 where we show the wide-field image of the binary mixture (Fig. 1 a) together with the corresponding super-resolved image of the same sample (Fig. 11 b). Despite the close proximity of most of the particles, the reconstruction of their position and size can be done very precisely to obtain a quantitatively sound particle size distribution (Fig. 1 c) that would be impossible to draw with the wide-field images. The same group also showed how a particular implementation of super-resolution microscopy (iPAINT: interface Point Accumulation for Imaging in Nanoscale Topography) permits nanoscale 
imaging of solid/liquid, liquid/liquid and liquid/air 285 at the nanoscale the morphology of complex coacervate core micelles in aqueous solutions, revealing a concentration-induced morphological transition of the micelles [13. Similar to PALM and 290 TORM, iPAINT is based on the localization of single isolated fluorescent molecules. This class of methods may benefit from the recent demonstration that combining them with multivariate statistical analysis opens the way to the 3D reconstruction 295 of nano-structures from 2D super-resolution projections 14 .

Another very interesting application is represented by the use of super-resolution imaging to inspect the interior of colloidal particles, in par- 300 ticular of polymer microgel particles 15-17. Microgel particles of PNIPAM, a polymer that exhibits a reversible volume phase transition at a well prescribed temperature of $32^{\circ}$, were studied with STORM with lateral and axial resolution of the or- 305 approach allowed reconstructing the internal structure of the microgel particles, and in particular their radial density profile, in different conditions, for instance, of temperature, solvent. The obtained re- 310 sults open the way to the tuning of the internal structure of the microgel particles that may be crucial in their optimization as drug delivery carriers.

Improving the accuracy of particle tracking algo- 315 rithms

One of the ways to improve the quantitativeness of optical microscopy experiments is based on increasing the precision and accuracy of the image processing. A paradigmatic example in this respect 320 is the need of obtaining very accurate estimates of the particle localization in tracking experiments, which can be used for several purposes. For example, following Perrin we can use the particle mean square displacements to determine diffusion coeffi- 325 cients or, more generally, the mechanical moduli of a soft material in which the colloidal particles have been embedded as probes, which is the realm of microrheology [18, 19.

Camera-based particle tracking determines the 330 position of a particle either by using Gaussian fitting or by calculating the intensity-based centroid, or by locating the local intensity maximum. The best performances of camera-based particle tracking using these approaches are currently of the or- 335 der of $10 \mathrm{KHz}$ and $3 \mathrm{~A}^{\circ}$ for the temporal and spa- tial resolution, respectively $21-23$ and it is likely that technological advances will improve these performances.

Another route toward improving particle tracking is based on making use of sophisticated image processing algorithms with standard instrumentation. A first example of application of this idea is represented by Bayesian localization microscopy, which allows extremely accurate particle localization to be extracted from wide-field images and, quite importantly, where labeling is obtained with standard fluorescent proteins 24. A Bayesian analysis of blinking and bleaching (3B analysis) leads to an impressive $50 \mathrm{~nm}$ resolution on a $4 \mathrm{~s}$ timescale, which is achieved by accurately modeling the blinking and bleaching of the fluorophores so to extract their most likely spatial distribution also when they are overlapping. This property represents an advantage over PALM- and STORM-like approaches, whose image reconstruction algorithm requires several tens of thousands of frames, each one of them obtained with non-overlapping fluorophores. Such advantage comes at the expense of a quite long computational time, about one day of computation time being needed for reconstructing a $50 \mu \mathrm{m}$ x $50 \mu \mathrm{m}$ region on a state-of-the-art desktop computer. However, it was shown in Ref. 25 how an improvement by a factor of 60 can be achieved by using cloud computing based on commercially available and accessible solutions.

A second example of analytical approach based on standard instrumentation is represented by super-resolution radial fluctuations (SRRF) microscopy 26], which combines considerations about the radial symmetry property of fluorophore emissions and a temporal fluctuation analysis of the frames to extract super-resolution images (at 1 frame per second and with a spatial resolution down to $60 \mathrm{~nm}$ ) with conventional fluorophores, low-intensity illumination and about 100 frames. Similar to the 3B analysis, also in SRRF a superresolution image is obtained without fluorophore detection and localization, which makes both of these techniques very appealing not only for use in the life science community in which they originated, but also for colloid scientists, which would surely benefit from the possibility of using conventional fluorophores in their experiments.

Finally, in Ref. 20, an accurate reconstruction of colloidal particles positions and sizes was achieved in standard confocal microscopy experiments by modeling the image formation process 

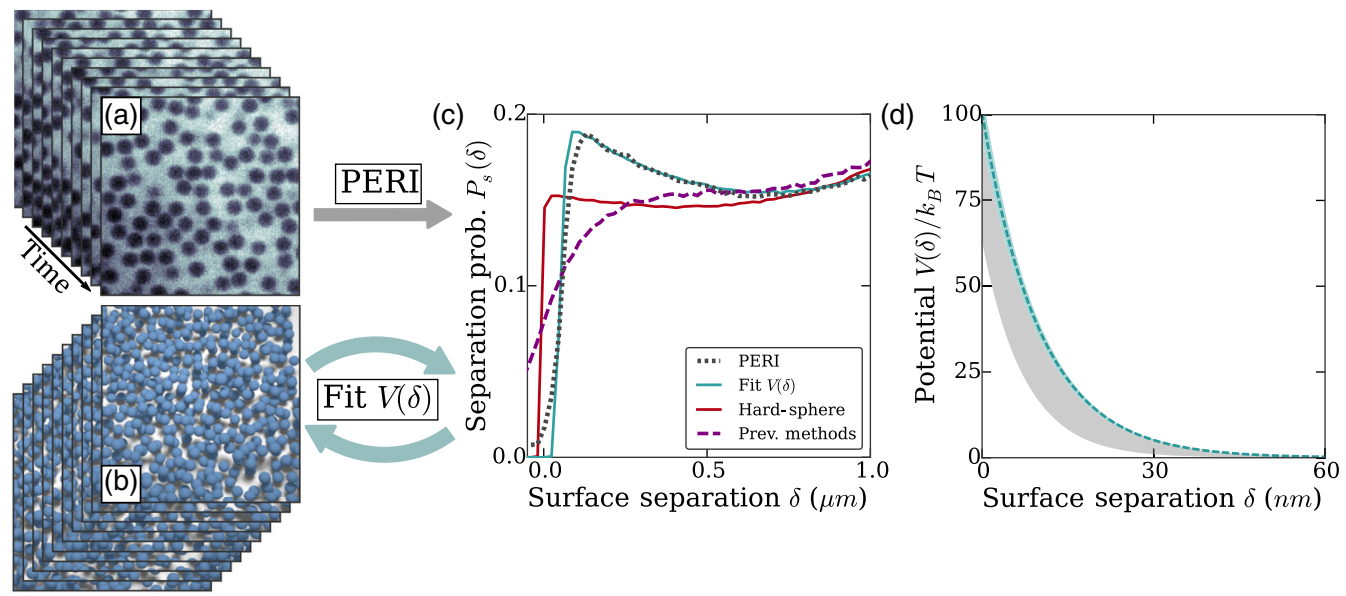

Figure 2: PERI is used to extract the interaction potential of a dilute suspension of $\sim 1200$ Brownian particles with diameter $1.3 \mu \mathrm{m}$. From the microscope images (a) the experimental probability $P_{s}(\delta)$ of finding a pair of particles with surface-to-surface separation $\delta$ is extracted and shown in (c) as a gray dashed line. In panel (c), we also show the best fit (solid cyan line) of the experimental data, which was obtained by running molecular dynamics simulations (b) and iteratively update the interaction potential $V(\delta)$ to match the experimental data. For comparison, the results obtained with a simple hard-sphere potential (red line) and with a standard particle-tracking approach (purple line) are also shown in (c). From the best-fit simulation, the interparticle potential is extracted (d). The shaded bands show the uncertainty in the potential, with the teal band describing uncertainty in the fit and the gray band the uncertainty due to systematic errors. Reprinted under CC BY 4.0 license from Ref. 20]. DOI:10.1103/PhysRevX.7.041007

and fitting the model parameters to the experimental data. This approach, termed parameter extraction from reconstructed images (PERI), was emonstrated in a proof-of-principle experiment in 36 which both the radius and position of $1.3 \mu \mathrm{m}$ particles were determined to within $3 \mathrm{~nm}$ and, by using 1200 particles, it was possible to reconstruct the repulsive interaction potential between the particles (Fig. 2). Once again at the expense of ${ }_{370}$ a very long computational time, a reconstructive generative model approach such as PERI outperforms heuristic approaches for tracking [27, such as the ones based on intensity centroids or on local fitting of the image intensity maxima to a Gaus- 37 sian function, providing a minimally-biased though computationally-intensive tool for particle tracking 28.

\section{Digital Fourier Microscopy of colloids: quantitative microscopy flirts with light scattering}

A notable dichotomy in modern colloid science is the one occurring between real- and reciprocal- 385 space sample observations. For years, optical microscopes have been used to perform real-space experiments, building on the fact that microscope movies capture the position of the colloidal particles in space and time. At the other extreme, we find light scattering methods that are based on experimental schemes to capture a far-field, reciprocalspace version of the same process. Of course, whether the 'glasses' used to watch the movie are built to probe the sample in real- or in reciprocalspace depends on the needs, skills and tools that are available to the experimenter. After the invention of the laser, scattering methods have dominated for long time the colloidal science laboratories, especially when small-sized, sub-diffraction particles were to be characterized. Recent advances, some of which are discussed in Section 3, slowly subtracted users to light scattering methods, with the main drive that watching in real space how a system looks gives more direct information. However, extracting this information to provide unbiased and robust statistical estimators of physical quantities is not a simple task. In addition, despite the technological and methodological progresses made, the signal-to-noise ratio, the resolution needs and the multiple-scattering limitations still make real-space approaches very challenging for dense suspensions of small colloidal particles. This is true in particular when the optical contrast of the single particles is too small, which affects the signal to noise ratio, or 
too large, which easily causes multiple scattering.

While these limit cases can still be safely managed with light scattering techniques 29], it was proposed in Ref. 30 that one can perform light scattering experiments on colloidal samples from the analysis of real-space movies. This approach, ermed Differential Dynamic Microscopy (DDM), is based on combining image subtractions and spatial Fourier transforms to obtain a characterization of the static and dynamic scattering signals from the sample in the reciprocal space $\left(q_{x}, q_{y}\right)$. With DDM, the characterization of the sample dynamics is calibration-free and can be almost automated. By contrast, static scattering information can be extracted with some additional calibration steps. Readers interested in the detail of this procedure, and in general in all the steps of the DDM analysis, can refer to the original papers [30, 31] or to recent reviews 32,33 . Another recent review article 34 describes DDM in the framework of Digital Fourier Microscopy (DFM) techniques, which have in common that, under proper conditions, they recover scattering information from a digital Fourier transform of real-space images.

A first notable feature of DDM is that it can be implemented with a variety of imaging contrast mechanisms that can operate in various conditions of signal-to-noise ratio, resolution needs and multiple scattering. Since its introduction about ten years ago, DDM was used to study colloids in bright-field [35-41, phase-contrast 42, dark-field 43, 44 and depolarized 45 microscopy. It has also been demonstrated with fluorescent particles in wide-field [36, 46, 47, confocal [48, 49] and lightsheet [50, 51] configurations.

Second, the requirements of DDM in terms of signal-to-noise ratio, resolution and absence of ${ }_{450}$ multiple-scattering are way less severe than for realspace tracking experiments, because in DDM: (i) particle localization is not needed and the signal of interest can be even considerably (e.g. a factor in 52 ) smaller than the noise, (ii) bright-field DDM is intrinsically more insensitive to multiple scattering than far-field scattering because the use of partially coherent light permits using very thin samples (of the order and below $100 \mu \mathrm{m}$ ) and also strongly reduces the effective thickness of the sam- ${ }_{460}$ ple and the effects of multiple scattering.

It was recently shown that DDM can be used for performing microrheology experiments [53, 54. DDM-microrheology (DDM- $\mu \mathrm{r}$ ) is an extension of the original Perrin experiment in at least two re- 465

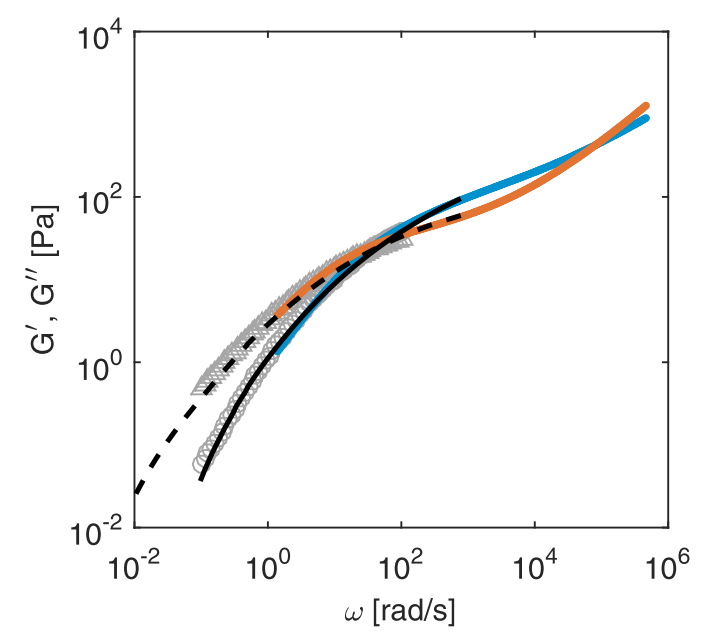

Figure 3: Comparison of the viscoelastic moduli $\mathrm{G}$ and $\mathrm{G}$ of a $2 \%$ PEO polymer solution in water, obtained with different methods. Gray circles (triangles): $G(G)$ obtained with a rheometer; continuous blue (orange) line: $G(G)$ obtained with Diffusing Wave Spectroscopy using $500 \mathrm{~nm}$ tracers; black continuous (dashed) line: $G(G)$ obtained with DDM microrheology (weighted average of the results of 100 $\mathrm{nm}$ and $500 \mathrm{~nm}$ tracers). Reprinted figure with permission from Ref. 53 Copyright (2017) by the American Physical Society.

spects: (i) it determines the particle mean square displacement in a host medium not from the tracks of individual particles but from the study of the intensity fluctuations associated to their motion; (ii) in the original spirit of microrheology 18 , it is based on a generalized Stokes-Einstein relation, which converts the mean square displacement of the tracer particles in the frequency-dependent storage $\left(G^{\prime}\right)$ and loss (G") moduli. The results obtained in Refs. [53, 54] show independently and very convincingly that DDM can obtain high quality microrheology data bypassing two of the major limitations of particle tracking, which are (i) the selection of the suitable reconstructed particle trajectories and (ii) the need of using colloidal tracers with a sufficiently large optical contrast. In addition, in Ref. 53 an optimization-based method is introduced that grants a calibration-free DDM- $\mu$ r experiment, which represents an additional advantage over particle tracking microrheology. The performance of DDM- $\mu$ r can be appreciated by inspecting Fig. 3. where the storage and loss moduli of a $2 \%$ PEO polymer solution in water obtained with different methods are shown. The DDM results (black dashed and continuous lines) are found in excellent 
agreement both at small frequencies with mechan- 515 ical rheometry (gray circles and triangles) and at large ones with Diffusing Wave Spectroscopy microrheology (orange and cyan continuous lines).

Interestingly, DDM was also used for accurate microrheology experiments probing both the trans- 520 lational and the rotational degrees of freedom of embedded tracer particles [53] and also in optically dense fluids [54, in both cases outperforming particle tracking.

\section{Poking while watching: quantitative mi- croscopy meets rheology}

The mechanical properties of amorphous materi- 530 als such as glasses (polymeric, metallic, and coloidal), gels (polymeric and colloidal), foams, or emulsions are not yet fully understood and are the subject of an intense research activity 55. When subjected to an external action, these materials ini- 535 tially deform in a reversible, elastic fashion. By contrast, a stress that is sufficiently large in amplitude or long in duration may result in plasticity and flow, similar to a viscous liquid. Yielding of the material and the subsequent plastic flow are ${ }_{540}$ determined by a series of microscopic events that eventually may lead to fracture and whose nature and properties have not yet been fully elucidated 56, 57.

Theoretically, fracture and plasticity in crys- 545 talline solids are well understood, with defects in the crystalline lattice (dislocations) playing a key role 58. By contrast, understanding the microscopic origin of yield and failure in amorphous solids is certainly far from complete. Most of the diffi- 550 culty resides in the absence of a symmetric structure, which could serve as reference to detect defects. During the last years, important progresses have been made also for amorphous materials. Theoretical work and simulation have suggested that ${ }_{555}$ plastic deformation results from the microscopic occurrence of shear transformations, local rearrangements involving few particles (of the order of 5-10) in well-defined regions, the so-called shear transformation zones (STZ) or 'soft spots' [59]. Such events originate anisotropic stresses (strains) known

510 as Eshelby stresses (strains), anisotropic perturbations with a characteristic quadrupolar spatial correlation, which perturb the otherwise isotropic surrounding elastic medium $[60-62$. Simulation also 565 shows that STZ manifest themselves in sheared liq- uids, seemingly suggesting that they are not peculiar to plastic deformation 63.

How far STZ can account for the failure of the wide variety of amorphous systems exhibiting similar mechanical failure modes is not yet established. In particular, the role of thermal noise has not been addressed in a systematic manner. This, however, is essential to bridge the gap from molecular to granular systems, including the intermediate soft glassy materials (SGM), all of these systems exhibiting similar failure modes.

As far as experiments are concerned, imaging atoms or molecules under mechanical stress is actually beyond reach since the constituents are too small and move too quickly. This is why during the last about fifteen years sheared colloidal gels, glasses and crystals have been widely investigated with quantitative microscopy. Much remains to be done, despite the relevant advances made. For instance, recent experiments with hard-sphere colloids show that STZ surprisingly exist not only in deformed glasses 64 but also in undeformed glasses 65) and defective crystals 66.

The striking observation of Eshelby strains in quiescent colloidal glasses, reported in 65 , is well described in Fig. 4, where we show the results of quantitative confocal microscopy experiments in which roughly 50000 monodisperse hard-spheres belonging to a colloidal glass are tracked in $3 D$ in the absence of an externally applied strain. While in the presence of an external shear the authors observe STZ that exhibit a bias in the shear strain direction and, in turn, contribute to the macroscopic deformation, the results in Fig. 4 show that STZ are also present in quiescent glasses, as they are activated by the thermal energy. However, they are weaker than the deformed case and they do not contribute to the macroscopic strain because there is no coupling with an external force field. If the glass is deformed above the yield point, some of the STZ do not relax back to the undeformed state contributing to an irreversible plastic deformation. Forcing the material back to an overall zero macroscopic strain causes the emergence of inclusions made of few particles that cancel out the strain contribution of the irreversible STZ.

It should be noted that rheo-microscopy (i.e. confocal microscopy under shear) experiments have been very popular to investigate, among several other phenomena, the structure 67 and the slip 68 of colloidal gels under steady shear. Similarly, when used on colloidal glasses, they have been pro- 
(1)

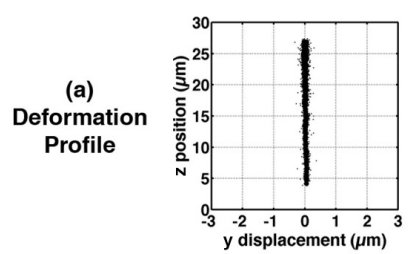

(b)

Highest

Strain

Particles

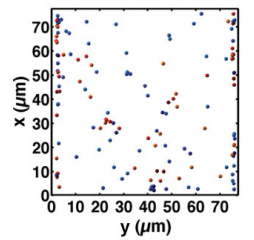

(c)
Spatial
orrelation
of Local
Strain

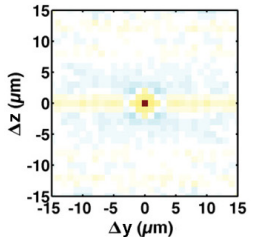

(2)
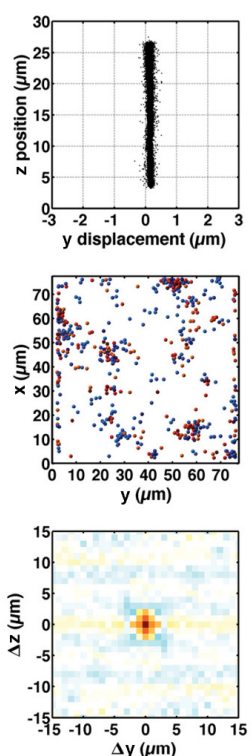

(3)
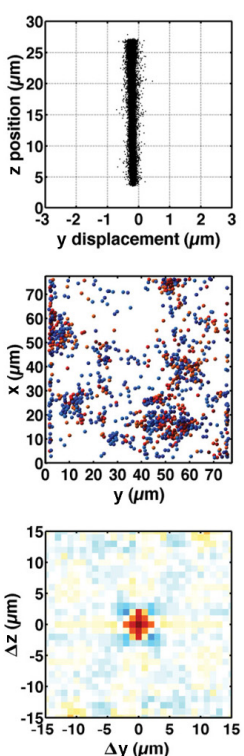

(4)
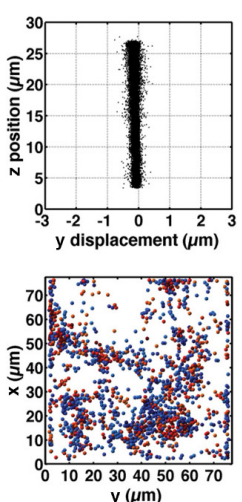

$\mathrm{y}(\mu \mathrm{m})$

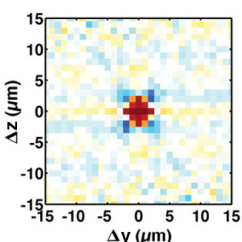

(5)
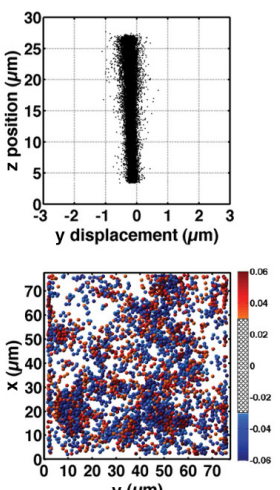

$(\mu \mathrm{m})$

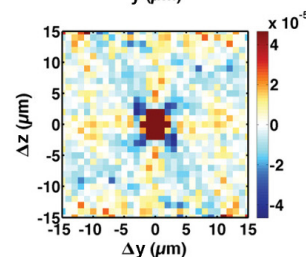

Figure 4: Evolution of strain and strain correlations in a quiescent colloidal glass at different time intervals. The deformation profiles (a) show broadening but no deformation. Row (b) shows top-view reconstructions showing only those particles with individual strain $\left|\varepsilon_{y z}\right|>0.03$, colored according to their strain. At any time, there are equal numbers of high positive (red) and negative (blue) particles. Row (c) shows the spatial $y-z$ plane strain correlations of $\varepsilon_{y z}$, showing the evolution of the fourfold Eshelby signature. Reprinted figure with permission from Ref. 65] Copyright (2016) by the American Physical Society.

viding useful information, for instance, on shear banding [69], shear concentration coupling [70] and creep [71]. In addition, they have been also used to probe the effect of the stress overshoot during startup shear $72-74$ and of a sudden cessation of shear 75. It would be interesting to use smaller collloidal particles to investigate how an increase of thermal noise would impact on the coupling between rheology, structure and dynamics. A possible way to overcome the limitations of real space approaches in tracking a crowded collection of small particles may be offered by Differential Dynamic Microscopy, which was described in Section 4

Before ending this Section, I would like to con- 605 sider polycrystals, an intermediate case between crystals and amorphous solids. Polycrystals are made of crystalline domains (grains) that are separated by grain boundaries, which are defect surfaces for 3D polycrystals or lines for polycrys- ${ }^{610}$ talline monolayers. The authors of Ref. [76] performed a very thorough quantitative microscopy study in which oscillatory shear deformations were applied with a magnetically oscillating microdisk to polycrystalline monolayers of soft repulsive colloids 615 trapped at a fluid interface. At the same time, an optical microscope was used to image the structure of the polycrystal at various distances from the edge of the disk.

The potential of this kind of experiments can be appreciated by inspecting Fig. 5in which a Voronoi reconstruction of a $\phi=13.5 \%$ monolayer is shown before shear is applied (Fig. 5a). The color in Fig. 5 a encodes the number of sides of the polygon. Hexagons (gray) are the majority but pentagons (yellow), heptagons (cyan) and octagons (orange) are also observed. As an oscillatory flow of the microdisk (dark gray with two holes) is applied with amplitude $\theta=10^{\circ}$ and frequency $\omega=0.1 \mathrm{~Hz}$, the defects start to move with spatially heterogeneous and temporally intermittent dynamics (Fig. 5b) and to interact (see for instance the annihilation of a pentagon and an hexagon from $t=20 \mathrm{~s}$ to $t=70$ s). Panels (c) and (d) show the phase angle of the orientational bond order parameter $\psi_{6}=\sum_{m} e^{6 i \theta_{m}}$ immediately before (c) and 20 min after the application of the shear. Color in panels (c) and (d) corresponds to the orientation of a single grain, which shows how the application of a moderate shear amplitude increases the quality of the crystalline order through defect annihilation. For larger shear am- 

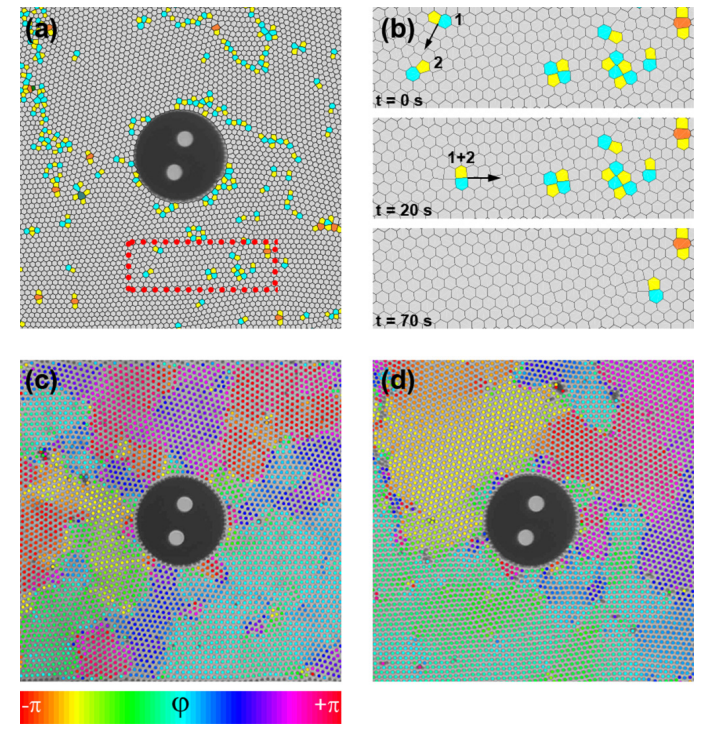

Figure 5: (a) Voronoi diagram of a colloidal monolayer with $\phi=13.5 \%$ and (b) time evolution of the region enclosed by the red rectangle. The gray cells are hexagonal, whereas the colored cells denote the local defects of the structure coming from particles with different numbers of neighbors, e.g., 5 (yellow) and 7 (cyan). (cd) Phase angle of the local $\psi_{6}$ function for a monolayers sheared at $\theta=10^{\circ}, \omega=0.1$ $\mathrm{Hz}$ for $20 \mathrm{~min}$. (c) Initial and (d) final state. Reprinted figure with permission from Ref. [76] Copyright (2017) by 660 the American Physical Society.

plitudes (data not shown), a critical strain exists above which the monolayer becomes fluid-like.

\section{Conclusions and Outlook}

In this short review, I have tried to give a personal overview of a few hot spots in the use of quantitative optical microscopy for the study of colloidal system. Of course, several at least equally im- ${ }^{670}$ portant studies were not mentioned here for space limitations. For instance, there is an increasingly large activity on the sedimentation kinetics and equilibrium of active colloids that would fit per- ${ }^{675}$ fectly within the scope of the present article but was not discussed here. Similar comments hold for other very active areas such as Digital Holographic Microscopy, for microscopy combined with ${ }^{680}$ optical/magnetic tweezers and for many others that I will not even mention here.

The cases presented here are, to some degree at least, ideal continuation of the original work of Perrin, in which the microscope was not used as a mysterious box for qualitative observation but rather a

very sophisticated instrument for quantitative and precise estimates to be compared with theoretical Courier Corporation, 2013.

[2] A. Einstein, Über die von der molekularkinetischen theorie der wärme geforderte bewegung von in ruhenden flüssigkeiten suspendierten teilchen, Ann. Phys. 322 (8) (1905) 549-560.

[3] J. C. Crocker, D. G. Grier, Methods of digital video microscopy for colloidal studies, J. Colloid Interface Sci. 179 (1) (1996) 298-310.

[4] E. R. Weeks, Microscopy of soft materials, Experimental and Computational Methods in Soft Condensed Matter.

[5] T. Lee, B. Senyuk, R. P. Trivedi, I. I. Smalyukh, Optical microscopy of soft matter systems, Fluids, Colloids and Soft Materials: An Introduction to Soft Matter Physics (2016) 165-185.

[6] S. W. Hell, J. Wichmann, Breaking the diffraction resolution limit by stimulated emission: stimulatedemission-depletion fluorescence microscopy, Opt. Lett. 19 (11) (1994) 780-782. 
[7] E. Betzig, G. H. Patterson, R. Sougrat, O. W. Lindwasser, S. Olenych, J. S. Bonifacino, M. W. Davidson, 755 J. Lippincott-Schwartz, H. F. Hess, Imaging intracellular fluorescent proteins at nanometer resolution, Science 313 (5793) (2006) 1642-1645.

[8] M. J. Rust, M. Bates, X. Zhuang, Sub-diffraction-limit imaging by stochastic optical reconstruction microscopy 760 (storm), Nat. Methods 3 (10) (2006) 793.

[9] B. Harke, C. K. Ullal, J. Keller, S. W. Hell, Threedimensional nanoscopy of colloidal crystals, Nano Lett. 8 (5) (2008) 1309-1313.

[10] M. A. Lauterbach, C. K. Ullal, V. Westphal, S. W. Hell, 765 Dynamic imaging of colloidal-crystal nanostructures at 200 frames per second, Langmuir 26 (18) (2010) 1440014404.

[11] A. Aloi, A. Vargas Jentzsch, N. Vilanova, L. Albertazzi, E. Meijer, I. K. Voets, Imaging nanostructures 770 by single-molecule localization microscopy in organic solvents, J. Am. Chem. Soc. 138 (9) (2016) 2953-2956.

[12] A. Aloi, N. Vilanova, L. Albertazzi, I. Voets, ipaint: a general approach tailored to image the topology of interfaces with nanometer resolution, Nanoscale 8 (16) 775 (2016) 8712-8716.

[13] A. Aloi, C. Guibert, L. L. Olijve, I. K. Voets, Morphological evolution of complex coacervate core micelles revealed by ipaint microscopy, Polymer 107 (2016) 450455.

[14] D. Salas, A. Le Gall, J.-B. Fiche, A. Valeri, Y. Ke, P. Bron, G. Bellot, M. Nollmann, Angular reconstitution-based $3 \mathrm{~d}$ reconstructions of nanomolecular structures from superresolution light-microscopy images, Proceedings of the National Academy of Sciences 785 114 (35) (2017) 9273-9278.

15] G. M. Conley, S. Nöjd, M. Braibanti, P. Schurtenberger, F. Scheffold, Superresolution microscopy of the volume phase transition of pnipam microgels, Colloids Surf., A 499 (2016) 18-23.

[16] A. P. Gelissen, A. Oppermann, T. Caumanns, P. Hebbeker, S. K. Turnhoff, R. Tiwari, S. Eisold, U. Simon, Y. Lu, J. Mayer, et al., 3d structures of responsive nanocompartmentalized microgels, Nano Lett. 16 (11) (2016) 7295-7301.

[17] S. Bergmann, O. Wrede, T. Huser, T. Hellweg, Superresolution optical microscopy resolves network morphology of smart colloidal microgels, Physical Chemistry Chemical Physics 20 (7) (2018) 5074-5083.

735 [18] T. G. Mason, D. Weitz, Optical measurements of 800 frequency-dependent linear viscoelastic moduli of complex fluids, Phys. Rev. Lett. 74 (7) (1995) 1250.

[19] E. M. Furst, T. M. Squires, Microrheology, Oxford University Press, 2017.

740 [20] M. Bierbaum, B. D. Leahy, A. A. Alemi, I. Cohen, J. P. 805 Sethna, Light microscopy at maximal precision, Phys. Rev. X 7 (4) (2017) 041007.

[21] G. M. Gibson, J. Leach, S. Keen, A. J. Wright, M. J. Padgett, Measuring the accuracy of particle position and force in optical tweezers using high-speed video mi- 810 croscopy, Opt. Express 16 (19) (2008) 14561-14570.

[22] O. Otto, F. Czerwinski, J. L. Gornall, G. Stober, L. B. Oddershede, R. Seidel, U. F. Keyser, Real-time particle tracking at 10,000 fps using optical fiber illumination, Opt. Express 18 (22) (2010) 22722-22733.

[23] A. Huhle, D. Klaue, H. Brutzer, P. Daldrop, S. Joo, O. Otto, U. F. Keyser, R. Seidel, Camera-based threedimensional real-time particle tracking at $\mathrm{khz}$ rates and ångström accuracy, Nat. Commun. 6 (2015) 5885.

[24] S. Cox, E. Rosten, J. Monypenny, T. JovanovicTalisman, D. T. Burnette, J. Lippincott-Schwartz, G. E. Jones, R. Heintzmann, Bayesian localization microscopy reveals nanoscale podosome dynamics, Nature methods 9 (2) (2012) 195.

[25] Y. S. Hu, X. Nan, P. Sengupta, J. LippincottSchwartz, H. Cang, Accelerating 3b single-molecule super-resolution microscopy with cloud computing, Nature methods 10 (2) (2013) 96.

[26] N. Gustafsson, S. Culley, G. Ashdown, D. M. Owen, P. M. Pereira, R. Henriques, Fast live-cell conventional fluorophore nanoscopy with imagej through superresolution radial fluctuations, Nature communications 7 (2016) 12471.

[27] N. Chenouard, I. Smal, F. De Chaumont, M. Maška, I. F. Sbalzarini, Y. Gong, J. Cardinale, C. Carthel, S. Coraluppi, M. Winter, et al., Objective comparison of particle tracking methods, Nature methods 11 (3) (2014) 281

[28] B. D. Leahy, M. Bierbaum, J. Sethna, I. Cohen, Biases in particle localization algorithms, arXiv preprint arXiv:1801.03581.

[29] F. Scheffold, R. Cerbino, New trends in light scattering, Current Opinion in Colloid \& Interface Science 12 (1) (2007) 50-57.

[30] R. Cerbino, V. Trappe, Differential dynamic microscopy: probing wave vector dependent dynamics with a microscope, Phys. Rev. Lett. 100 (18) (2008) 188102.

[31] F. Giavazzi, D. Brogioli, V. Trappe, T. Bellini, R. Cerbino, Scattering information obtained by optical microscopy: differential dynamic microscopy and beyond, Physical Review E 80 (3) (2009) 031403.

[32] R. Cerbino, A. Vailati, Near-field scattering techniques: Novel instrumentation and results from time and spatially resolved investigations of soft matter systems, Current Opinion in Colloid \& Interface Science 14 (6) (2009) 416-425.

[33] R. Cerbino, P. Cicuta, Perspective: differential dynamic microscopy extracts multi-scale activity in complex fluids and biological systems, The Journal of Chemical Physics 147 (11) (2017) 110901.

[34] F. Giavazzi, R. Cerbino, Digital fourier microscopy for soft matter dynamics, J. Opt. 16 (8) (2014) 083001.

[35] F. Ferri, A. DAngelo, M. Lee, A. Lotti, M. Pigazzini, K. Singh, R. Cerbino, Kinetics of colloidal fractal aggregation by differential dynamic microscopy, The European Physical Journal Special Topics 199 (1) (2011) $139-148$.

[36] K. He, M. Spannuth, J. C. Conrad, R. Krishnamoorti, Diffusive dynamics of nanoparticles in aqueous dispersions, Soft Matter 8 (47) (2012) 11933-11938.

[37] K. He, F. Babaye Khorasani, S. T. Retterer, D. K. Thomas, J. C. Conrad, R. Krishnamoorti, Diffusive dynamics of nanoparticles in arrays of nanoposts, ACS Nano 7 (6) (2013) 5122-5130.

[38] A. Wittmeier, A. Leeth Holterhoff, J. Johnson, J. G. Gibbs, Rotational analysis of spherical, optically anisotropic janus particles by dynamic microscopy, Langmuir 31 (38) (2015) 10402-10410.

[39] F. Giavazzi, G. Savorana, A. Vailati, R. Cerbino, Structure and dynamics of concentration fluctuations in a non-equilibrium dense colloidal suspension, Soft Matter 12 (31) (2016) 6588-6600. 
[40] D. Germain, M. Leocmach, T. Gibaud, Differential dynamic microscopy to characterize brownian motion and 885 bacteria motility, Am. J. Phys 84 (3) (2016) 202-210.

[41] M. S. Safari, R. Poling-Skutvik, P. G. Vekilov, J. C. Conrad, Differential dynamic microscopy of bidisperse colloidal suspensions, npj Microgravity 3 (1) (2017) 21.

[42] M. Reufer, V. A. Martinez, P. Schurtenberger, W. C. 890 Poon, Differential dynamic microscopy for anisotropic colloidal dynamics, Langmuir 28 (10) (2012) 4618-4624.

[43] A. V. Bayles, T. M. Squires, M. E. Helgeson, Darkfield differential dynamic microscopy, Soft Matter 12 (8) (2016) 2440-2452.

[44] R. Cerbino, D. Piotti, M. Buscaglia, F. Giavazzi, Dark field differential dynamic microscopy enables accurate characterization of the roto-translational dynamics of bacteria and colloidal clusters, J. Phys.: Condens. Matter 30 (2) (2017) 025901.

[45] F. Giavazzi, C. Haro-Pérez, R. Cerbino, Simultaneous characterization of rotational and translational diffusion of optically anisotropic particles by optical microscopy, J. Phys.: Condens. Matter 28 (19) (2016) 195201.

[46] J. D. C. Jacob, K. He, S. T. Retterer, R. Krishnamoorti, J. C. Conrad, Diffusive dynamics of nanoparticles in ultra-confined media, Soft Matter 11 (38) (2015) 75157524

[47] N. Shokeen, C. Issa, A. Mukhopadhyay, Compari- 910 son of nanoparticle diffusion using fluorescence correlation spectroscopy and differential dynamic microscopy within concentrated polymer solutions, Appl. Phys. Lett. 111 (26) (2017) 263703.

[48] P. J. Lu, F. Giavazzi, T. E. Angelini, E. Zaccarelli, 915 F. Jargstorff, A. B. Schofield, J. N. Wilking, M. B. Romanowsky, D. A. Weitz, R. Cerbino, Characterizing concentrated, multiply scattering, and actively driven fluorescent systems with confocal differential dynamic microscopy, Phys. Rev. Lett. 108 (21) (2012) 218103.

[49] T. Sentjabrskaja, E. Zaccarelli, C. De Michele, F. Sciortino, P. Tartaglia, T. Voigtmann, S. U. Egelhaaf, M. Laurati, Anomalous dynamics of intruders in a crowded environment of mobile obstacles, Nat. Commun. 7 (2016) 11133.

[50] D. M. Wulstein, K. E. Regan, R. M. RobertsonAnderson, R. McGorty, Light-sheet microscopy with digital fourier analysis measures transport properties over large field-of-view, Opt. Express 24 (18) (2016) 20881-20894.

[51] D. M. Wulstein, R. McGorty, Point-spread function engineering enhances digital fourier microscopy, Opt. Lett. 42 (22) (2017) 4603-4606.

[52] F. Giavazzi, P. Edera, P. J. Lu, R. Cerbino, Image windowing mitigates edge effects in differential dynamic 935 microscopy, The European Physical Journal E 40 (11) (2017) 97.

[53] P. Edera, D. Bergamini, V. Trappe, F. Giavazzi, R. Cerbino, Differential dynamic microscopy microrheology of soft materials: A tracking-free determination of 940 the frequency-dependent loss and storage moduli, Physical Review Materials 1 (7) (2017) 073804.

[54] A. V. Bayles, T. M. Squires, M. E. Helgeson, Probe microrheology without particle tracking by differential dynamic microscopy, Rheol. Acta 56 (11) (2017) 863- 945 869.

[55] D. T. Chen, Q. Wen, P. A. Janmey, J. C. Crocker, A. G. Yodh, Rheology of soft materials, Annu. Rev. Condens.
Matter Phys.

[56] D. Bonn, M. M. Denn, L. Berthier, T. Divoux, S. Manneville, Yield stress materials in soft condensed matter, Rev. Mod. Phys. 89 (3) (2017) 035005.

[57] P. Coussot, Slow flows of yield stress fluids: yielding liquids or flowing solids?, Rheol. Acta (2017) 1-14.

[58] R. M. Christensen, The theory of materials failure, Oxford University Press, 2013.

[59] A. Nicolas, E. E. Ferrero, K. Martens, J.-L. Barrat, Deformation and flow of amorphous solids: a review of mesoscale elastoplastic models, arXiv preprint arXiv:1708.09194.

[60] M. L. Falk, J. S. Langer, Deformation and failure of amorphous, solidlike materials, Annu. Rev. Condens. Matter Phys. 2 (1) (2011) 353-373.

[61] J.-L. Barrat, A. Lemaitre, Heterogeneities in amorphous systems under shear, Dynamical heterogeneities in glasses, colloids, and granular media 150 (2011) 264

[62] T. Voigtmann, Nonlinear glassy rheology, Current Opinion in Colloid \& Interface Science 19 (6) (2014) 549-560.

905 [63] J. Chattoraj, A. Lemaitre, Elastic signature of flow events in supercooled liquids under shear, Phys. Rev. Lett. 111 (6) (2013) 066001.

[64] P. Schall, D. A. Weitz, F. Spaepen, Structural rearrangements that govern flow in colloidal glasses, Science 318 (5858) (2007) 1895-1899.

[65] K. Jensen, D. A. Weitz, F. Spaepen, Local shear transformations in deformed and quiescent hard-sphere colloidal glasses, Physical Review E 90 (4) (2014) 042305.

[66] N. Y. Lin, M. Bierbaum, P. Schall, J. P. Sethna, I. Cohen, Measuring nonlinear stresses generated by defects in 3d colloidal crystals, Nat. Mater. 15 (11) (2016) 1172.

[67] N. Koumakis, E. Moghimi, R. Besseling, W. C. Poon, J. F. Brady, G. Petekidis, Tuning colloidal gels by shear, Soft Matter 11 (23) (2015) 4640-4648.

[68] P. Ballesta, N. Koumakis, R. Besseling, W. C. Poon, G. Petekidis, Slip of gels in colloid-polymer mixtures under shear, Soft Matter 9 (12) (2013) 3237-3245.

[69] P. Ballesta, R. Besseling, L. Isa, G. Petekidis, W. Poon, Slip and flow of hard-sphere colloidal glasses, Phys. Rev. Lett. 101 (25) (2008) 258301.

[70] R. Besseling, L. Isa, P. Ballesta, G. Petekidis, M. Cates, W. Poon, Shear banding and flow-concentration coupling in colloidal glasses, Phys. Rev. Lett. 105 (26) (2010) 268301

930 [71] T. Sentjabrskaja, P. Chaudhuri, M. Hermes, W. Poon, J. Horbach, S. Egelhaaf, M. Laurati, Creep and flow of glasses: strain response linked to the spatial distribution of dynamical heterogeneities, Sci. Rep. 5 (2015) 11884 .

[72] J. Zausch, J. Horbach, M. Laurati, S. U. Egelhaaf, J. M. Brader, T. Voigtmann, M. Fuchs, From equilibrium to steady state: the transient dynamics of colloidal liquids under shear, J. Phys.: Condens. Matter 20 (40) (2008) 404210.

[73] N. Koumakis, M. Laurati, S. Egelhaaf, J. Brady, G. Petekidis, Yielding of hard-sphere glasses during start-up shear, Phys. Rev. Lett. 108 (9) (2012) 098303.

[74] M. Laurati, P. Maßhoff, K. J. Mutch, S. U. Egelhaaf, A. Zaccone, Long-lived neighbors determine the rheological response of glasses, Phys. Rev. Lett. 118 (1) (2017) 018002.

[75] M. Ballauff, J. M. Brader, S. U. Egelhaaf, M. Fuchs, J. Horbach, N. Koumakis, M. Krüger, M. Laurati, K. J. 
Mutch, G. Petekidis, et al., Residual stresses in glasses,

950 Phys. Rev. Lett. 110 (21) (2013) 215701.
[76] I. Buttinoni, M. Steinacher, H. T. Spanke, J. Pokki, S. Bahmann, B. Nelson, G. Foffi, L. Isa, Colloidal polycrystalline monolayers under oscillatory shear, Physical Review E 95 (1) (2017) 012610. 
[15] G. M. Conley, S. N"ojd, M. Braibanti, P. Schurtenberger, F. Scheffold, Superresolution microscopy of the volume phase transition of pnipam microgels, Colloids Surf., A 499 (2016) 18-23.

* Using direct STochastic Optical Reconstruction Microscopy (dSTORM) the Authors perform one of the first studies of the interior of thermosensitive microgel particles with a lateral resolution of $30 \mathrm{~nm}$ and an axial resolution of $60 \mathrm{~nm}$.

[23] A. Huhle, D. Klaue, H. Brutzer, P. Daldrop, S. Joo, O. Otto, U. F. Keyser, R. Seidel, Camera-based three-dimensional real-time particle tracking at khz rates and angstr "om accuracy, Nat. Commun. 6 (2015) 5885.

** First article that brings the performances of real-time, camera-based multi-particle tracking to sensitivity levels close to the ones obtained with setups making use of quadrant-photodiode-based detection of single-particles.

[26] N. Gustafsson, S. Culley, G. Ashdown, D. M. Owen, P. M. Pereira, R. Henriques, Fast live-cell conventional fluorophore nanoscopy with imagej through super-resolution radial fluctuations, Nature communications 7 (2016) 12471.

** The Authors introduce and make available also as an Imagej plugin a novel superresolution algorithm that can be used with low-levels of illumination and with a variety of standard microscopy modes, including wide-field, confocal and TIRF imaging.

[50] D. M. Wulstein, K. E. Regan, R. M. Robertson, R. Anderson, R. McGorty, Light-sheet microscopy with $\square$ digital fourier analysis measures transport properties $\square$ over large fieldof-view, Opt. Express 24 (18) (2016) 20881-20894.

* The Authors combine Differential Dynamic Microscopy with light-sheet illumination, providing a very powerful probe of the dynamics with minimal photobleaching.

[66] N. Y. Lin, M. Bierbaum, P. Schall, J. P. Sethna, I. Cohen, Measuring nonlinear stresses generated by defects in 3d colloidal crystals, Nat. Mater. 15 (11) (2016) 1172. ** By using the SALSA (stress assessment from local structural anisotropy) technique, the Authors perform an unprecedented study of the stresses surrounding defects in a colloidal crystalline structure obtained with hard-spheres.

[67] N. Koumakis, E. Moghimi, R. Besseling, W. C. Poon, J. F. Brady, G. Petekidis, Tuning colloidal gels by shear, Soft Matter 11 (23) (2015) 4640-4648.

* Thorough study of the interplay between the structural and rheological properties of intermediate volume fraction colloidal gels submitted to a steady-state shear flow or to shear cessation. Simultaneous rheometry, confocal microscopy and simulations study the effect of varying the applied shear rate on the structure of the gel. Strong shearing favors stiffer and homogeneous gels, whereas soft shearing perturbations lead to weak and heterogenous gels.

[74] M. Laurati, P. Maßhoff, K. J. Mutch, S. U. Egelhaaf, A. Zaccone, Long-lived neighbors determine the rheological response of glasses, Phys. Rev. Lett. 118 (1) (2017) 018002.

* A combination of experiments and theoretical modeling unveils how the macroscopic transient rheological response of colloidal glasses is connected to the shear-induced microscopic structural evolution of the glass, and in particular to the number of longlived neighbors i.e. particles that remain nearest neighbors for a long time. 